\title{
Mixed micelles loaded with silybin-polyene phosphatidylcholine complex improve drug solubility
}

\author{
Rui-ling DUAN, Xun SUN, Jie LIU, Tao GONG* , Zhi-rong ZHANG*
}

Key Laboratory of Drug Targeting and Drug Delivery Systems, Ministry of Education, West China School of Pharmacy, Sichuan University, Chengdu 610041, China

\begin{abstract}
Aim: To prepare a novel formulation of phosphatidylcholine (PC)-bile salts (BS)-mixed micelles (MMs) loaded with silybin (SLB)-PC complex for parenteral applications.

Methods: SLB-PC-BS-MMs were prepared using the co-precipitation method. Differential scanning calorimetry (DSC) analysis was used to confirm the formation of the complex and several parameters were optimized to obtain a high quality formulation. The water-solubility, drug loading, particle size, zeta potential, morphology and in vivo properties of the SLB-PC-BS-MMs were determined.

Results: The solubility of SLB in water was increased from $40.83 \pm 1.18 \mu \mathrm{g} / \mathrm{mL}$ to $10.14 \pm 0.36 \mathrm{mg} / \mathrm{mL}$ with a high drug loading (DL) of $14.43 \% \pm 0.44 \%$ under optimized conditions. The SLB-PC-BS-MMs were observed by transmission electron microscopy (TEM) and scanning electron microscopy (SEM) and showed spherical shapes. The particle size and zeta potential, as measured by photon correlation spectroscopy (PCS), were about $30 \pm 4.8 \mathrm{~nm}$ and $-39 \pm 5.0 \mathrm{mV}$, respectively. In vivo studies showed that incorporation of the SLB-PC complex into PC-BS-MMs led to a prolonged circulation time of the drug.

Conclusion: This novel formulation appears to be a good candidate for drug substances that exhibit poor solubility for parenteral administration.
\end{abstract}

Keywords: silybin; bile salts; phospholipid complex; mixed micelles; solubility

Acta Pharmacologica Sinica (2011) 32: 108-115; doi: 10.1038/aps.2010.192; published online 20 Dec 2010

\section{Introduction}

The solubility of active pharmaceutical ingredients (APIs) has always been a concern for formulations because inadequate aqueous solubility may hamper the development of parenteral products and limit their bioavailability ${ }^{[1]}$. To solve this problem, several approaches have emerged to improve API solubility, such as salt formation ${ }^{[2]}$, crystal engineering ${ }^{[3]}$, nanosizing $^{[4]}$, lipid formulation ${ }^{[5]}$, cyclodextrin complexation ${ }^{[6]}$ and prodrug strategies ${ }^{[7]}$. Among these, we focused on the mixed micelle systems due to their synergistic properties, such as increased micelle stability and drug loading efficiency, which are superior to those of the individual components ${ }^{[8]}$.

In the current study, we selected silybin (SLB) as the model drug to validate the feasibility of mixed micelle systems. Silybin, a flavolignane that represents approximately $60 \%-70 \%$ of silymarin, is an antioxidant isolated from the fruits of milk thistle, Silybum marianum Gaertn. Interest in this agent has

\footnotetext{
* To whom correspondence should be addressed.

E-mail gongtaoy@126.com (Tao GONG); zrzzl@vip.sina.com (Zhi-rong ZHANG)

Received 2010-06-20 Accepted 2010-10-12
}

grown in recent years due to its wide array of beneficial pharmacological effects, including antioxidative, anti-lipid-peroxidative, antifibrotic, anti-inflammatory, immunomodulating and even liver regenerating effects ${ }^{[9-11]}$.

Despite the promising biological effects of SLB, its poor water solubility (about $40 \mu \mathrm{g} / \mathrm{mL}$ ) and oral bioavailability (about $0.73 \%$ ) in both rodents and humans, as reported by several researchers ${ }^{[12-15]}$, has restricted its clinical application. To improve this situation, there have been many contributions to the development of SLB formulations ${ }^{[15-17]}$. However, few have produced a marked effect, and a majority of them are based on oral drug delivery systems. Because no proper formulation of silybin has been approved for intravenous administration, our goal was to develop a novel mixed micelle system for its parenteral application that could dramatically improve its solubility.

Upon consideration of its physiological compatibility and solubilizing capacity, the phosphatidylcholine-bile saltsmixed micelle system (PC-BS-MM) seems to be a good candidate for intravenous drug administration ${ }^{[18,19]}$. Bile salts (BS), detergent-like chemicals produced by the liver and stored in the gall bladder, are able to solubilize phosphatidylcholine 
(PC) to a large extent, forming a clear mixed micellar solution that is able to solubilize substances with poor water solubil${ }^{i t y}{ }^{[20]}$. Previous studies have shown that the hemolytic effect of bile salts can be neutralized by solubilized phospholipids ${ }^{[21]}$. In addition, PC-BS-MMs can be locally and systemically tolerated with no embryotoxic, teratogenic or mutagenic effects ${ }^{[22]}$. Therefore, these PC-BS-MMs might be the ideal drug carrier of SLB for parenteral administration.

Despite the encouraging potential applications of PC-BSMMs mentioned above, preparing formulations of SLB with poor solubility enveloped in these carriers remains a challenge. Thus, it is expected that the SLB-PC complex, with changed solubility, would be better incorporated into PC-BSMMs.

To examine the potential of our model (PC-BS-MMs) as a novel delivery vehicle for water-insoluble drugs, the specific objectives of this work were (i) to formulate an SLB-PC complex in PC-BS-MMs for parenteral application by screening different formulation factors, (ii) to investigate the characteristics of the optimized SLB-PC-BS-MM formulation and (iii) to compare the pharmacokinetic parameters in vivo between the SLB-PC-BS-MMs and silybin- $N$-methylglucamine.

\section{Materials and methods}

Silybin (SLB) was purchased from Xi'an Sino-herb Bio-technology Co Ltd (Xi'an, china), purity 98.32\%. Polyene Phosphatidylcholine (PPC) was obtained from Lipoid GmbH (Germany), purity $94 \%$. Sodium deoxycholate (SDC) was supplied by Beijing Aoboxing Biotechnologies Co Ltd (Beijing, China). The other chemical reagents were of analytical grade or better.

Male Wistar rats were provided by the West China Experimental Animal Center of Sichuan University (China). The protocol was approved by the Institutional Animal Care and Use Committee of Sichuan University.

\section{Preparation of SLB-PPC complex}

The preparation of SLB-PPC complex was carried out as previously described ${ }^{[15]}$ with slight modifications. The complex was prepared with SLB and PPC at a molar ratio of 1:1. Weighed amounts of SLB and PPC were placed in a round bottom flask and dissolved in acetone. The mixture was then refluxed at $50{ }^{\circ} \mathrm{C}$ for $2 \mathrm{~h}$. After the acetone was evaporated off under vacuum (Büchi, R-144 rotary evaporator, Switzerland), the dried residues were collected and placed in desiccators.

\section{Structure validation of SLB-PPC complex by differential scanning calorimetry (DSC)}

DSC was performed on an EXSTAR 6000 thermogravimetric analyzer (EIKO, Japan), and a scan speed of $5{ }^{\circ} \mathrm{C}$ from 0 to $250{ }^{\circ} \mathrm{C}$ was employed. DSC thermograms of SLB, PPC, a physical mixture of SLB and PPC, and the SLB-PPC complex were compared to identify formation of the complex.

Preparation of PPC-BS-MMs loaded with the SLB-PPC complex (SLB-PPC-BS-MMs)

Because the SLB-PPC complex exhibited good solubility in anhydrous ethanol, the complex was easily loaded into PPCBS-MMs using the co-precipitation method ${ }^{[23]}$. In brief, the complex, PPC and sodium deoxycholate (SDC) at certain molar ratios were dissolved in anhydrous ethanol. A film was formed after evaporation of the organic solvent at $50{ }^{\circ} \mathrm{C}$. The resulting films were dispersed in double distilled water. To remove unsolubilized drug, the solutions were centrifuged at $16000 \times g$ (Allegra X-22R Centrifuge, F2402H rotor, Beckman Coulter Inc, CA, USA) for $10 \mathrm{~min}$. The supernatant contained the freshly formed mixed micelles. To increase the stability of the MMs, they were pre-frozen at $-40{ }^{\circ} \mathrm{C}$ for at least $4 \mathrm{~h}$ and then lyophilized under a vacuum for $24 \mathrm{~h}$.

\section{Determination of drug solubility and drug loading (DL\%) in different micelle systems}

The content of the SLB solubilized by MMs was determined by reverse-phase HPLC. SLB-PPC-SDC-MMs were diluted with methanol to a certain concentration and injected into an HPLC system. The stationary phase, a Kromasil C18 column (150 $\mathrm{mm} \times 4.6 \mathrm{~mm}, 5 \mu \mathrm{m})$, was kept at $35^{\circ} \mathrm{C}$. The mobile phase consisted of a mixture of acetonitrile and double distilled water (35:65) [ $\mathrm{pH}$ adjusted to 3-4 using 10\% phosphoric acid $(v / v)]$. The injection volume of the samples and standards was $20 \mu \mathrm{L}$. The flow rate was $1.0 \mathrm{~mL} / \mathrm{min}$ and the detection wavelength was $288 \mathrm{~nm}$.

The drug loading (DL\%) was calculated as follows:

$$
\mathrm{DL} \%=\frac{\text { Amount of drug in prescription }}{\begin{array}{c}
\text { Amount of } \\
\text { drug added }
\end{array}+\begin{array}{c}
\text { Amount of } \\
\text { excipent added }
\end{array}} \times 100 \%
$$

Determination of particle size and Zeta potential of SLB-PPCSDC-MMs

After suspending the freeze-dried SLB-PPC-SDC-MMs in double distilled water, photon correlation spectroscopy (PCS) (Malvern Zetasizer Nano ZS90, Malvern instruments Ltd, UK) was used to measure the particle size and zeta potential of the MMs. Each sample was then analyzed in triplicate.

Morphology of SLB-PPC-SDC-MMs determined by scanning electron microscopy (SEM) and transmission electron microscopy (TEM)

One drop of the diluted SLB-PPC-SDC-MMs was placed on a graphite surface. After being oven-dried, the sample was coated with gold using an ion sputter and examined with a conventional scanning electron microscope (JSM-5900LV, JEOL, Japan).

Before analysis by transmission electron microscopy, diluted samples were placed on copper grids with films and then stained with $2 \%(w / v)$ phosphotungstic acid; the excess staining solution was removed with filter paper. After being air dried, the films were observed with a transmission electron microscope (H-600, Hitachi, Japan).

\section{In vivo studies in rats}

Ten rats $(200 \pm 20 \mathrm{~g})$ were divided into 2 groups at random. 
Silybin-N-methylglucamine solution or SLB-PPC-SDC-MMs (corresponding to a dose of $80 \mathrm{mg} / \mathrm{kg}$ ) was injected into the tail vein of the rats of each group, respectively. After administration, blood was collected from the end of the tail at specific time points. Blood was immediately processed for plasma by centrifugation at $1500 \times g$ for $10 \mathrm{~min}$. The obtained plasma was stored at $-20{ }^{\circ} \mathrm{C}$ until analysis.

The plasma samples were prepared as follows to determine the SLB. One hundred microliters of internal standard (andrographolide: $200 \mu \mathrm{g} / \mathrm{mL}$ ) and $100 \mu \mathrm{L}$ of $0.1 \mathrm{~mol} / \mathrm{L}$ phosphate buffer ( $\mathrm{pH} 8.0$ ) were added to $100 \mu \mathrm{L}$ of plasma, followed by agitation for $30 \mathrm{~s}$. Then, $2.5 \mathrm{~mL}$ of absolute ether was added, and this mixture was shaken for $15 \mathrm{~min}$ and then centrifuged at $1500 \times g$ for $5 \mathrm{~min}$. Two milliliters of the organic phase was quantitatively transferred into clear centrifuging tubes and evaporated under nitrogen at $40{ }^{\circ} \mathrm{C}$. The residues were resuspended in $100 \mu \mathrm{L}$ of methanol and centrifuged at $9200 \times g$ for $10 \mathrm{~min}$. Next, $20 \mu \mathrm{L}$ of the supernatant was injected for HPLC analysis. The HPLC conditions were the same as the determination of drug solubility.

\section{Statistical analysis}

The results were analyzed by Drug and Statistics (DAS) software. Significance was tested by a two-tailed $t$-test and a value of $P<0.05$ was considered statistically significant.

\section{Results}

\section{Structure identification of SLB-PPC complex by DSC}

Differential scanning calorimetry (DSC) is a fast and reliable method to identify the formation of phospholipid complexes $^{[15,24]}$. Figure 1 shows the DSC thermograms of pure silybin (SLB), polyene phosphatidylcholine (PPC), the SLBPPC complex and a physical mixture of SLB and PPC.

The melting point of SLB was $136.5^{\circ} \mathrm{C}$. The DSC profile of

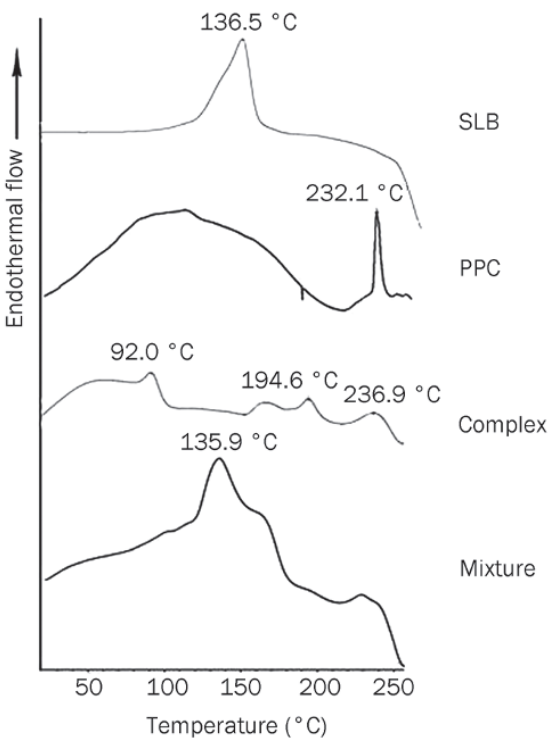

Figure 1. DSC thermograms of SLB, PPC, the SLB-PPC complex and a physical mixture of SLB and PPC.
PPC showed two different types of endothermal peaks. The first mild endothermal peak at approximately $109.4{ }^{\circ} \mathrm{C}$ was probably due to movement of the polar regions of the PPC molecule. The second endothermal peak at $232.1^{\circ} \mathrm{C}$ was very sharp, and its appearance was likely due to a phase transition from a gel to a liquid crystalline state. The non-polar hydrocarbon tail of PPC may melt during this phase, yielding a sharp peak.

The thermogram of a physical mixture of SLB and PPC was almost the overlap of each individual component, except for some slight differences. Specifically, the peak at $135.9^{\circ} \mathrm{C}$, which should be the melting point of SLB, was a little lower than that of pure SLB. The second peak of PPC disappeared, indicating that the PPC melted and the drug dissolved in the PPC at higher temperature. It is likely that a SLB-PPC complex was partially formed.

The thermogram of the SLB-PPC complex showed that the endothermal peaks of SLB and PPC had almost disappeared with the exception of a small peak at $236.9{ }^{\circ} \mathrm{C}$, which was probably due to a very small amount of unreacted PPC. Two more new peaks at 92.0 and $194.6{ }^{\circ} \mathrm{C}$ were present, indicating the formation of a SLB-PPC complex. The peak at $92.0^{\circ} \mathrm{C}$ was attributed to interactions of the polar groups of SLB and PPC in the form of hydrogen bonds, which occur through the $-\mathrm{OH}$ groups of the phenol rings of $\operatorname{SLB}^{[15,24]}$. Such interactions are supposed to be the dominant factor in the complexation process, as the peak at $92.0^{\circ} \mathrm{C}$ in the DSC curve absorbed more energy than the others. The peak at $194.6^{\circ} \mathrm{C}$, which absorbed less energy, might be associated with the changed spatial structure of the hydrocarbon chains.

Determination of drug solubility and drug loading (DL\%) in different micelle systems

The influence of the SDC concentration on SLB solubility in the mixed micelles and the drug loading efficiency is shown in Figure 2. Upon increasing the SDC concentration from 8 to 40 $\mathrm{mg} / \mathrm{mL}$, a significant increase in SLB solubility was observed, but a further increase in SDC concentration led to saturation. As for the drug loading efficiency, an enhanced SDC concentration (from 8 to $20 \mathrm{mg} / \mathrm{mL}$ ) led to an increased drug loading of up to $14.43 \%$. However, a continuous increase in SDC

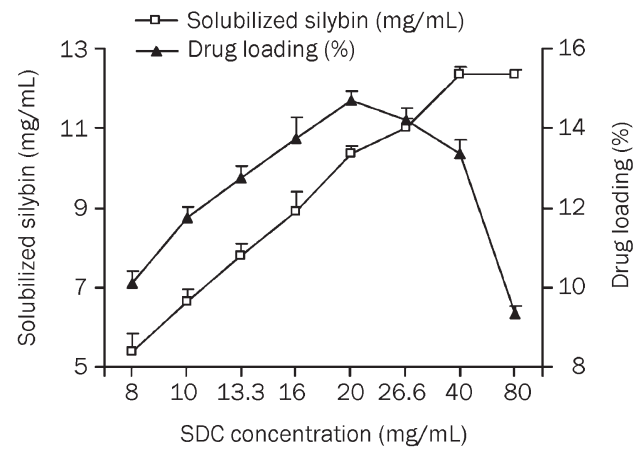

Figure 2. Influence of the SDC concentration on SLB solubility and drug loading at $10{ }^{\circ} \mathrm{C}$ (PPC conc, $\left.40 \mathrm{mg} / \mathrm{mL}\right)(n=3)$. 
concentration did not improve the solubilization efficiency. Therefore, an SDC concentration of $20 \mathrm{mg} / \mathrm{mL}$, at which concentration the PPC-SDC molar ratio was 1 and solubilized silybin could be up to $10.14 \pm 0.36 \mathrm{mg} / \mathrm{mL}$, was adopted for further investigations.

The influence of the PPC concentration on SLB solubility and drug loading efficiency was also investigated. As shown in Figure 3, SLB solubility was enhanced by incorporating increasing amounts of PPC in the MM system. After reaching a concentration of $60 \mathrm{mg} / \mathrm{mL}$, continuously increasing the PPC concentration resulted in a relatively stable solubilization of SLB and eventually decreased solubility. However, a further increase in the PPC amount (after $40 \mathrm{mg} / \mathrm{mL}$ ) led to a dramatic decrease in drug loading efficiency. Hence, to ensure higher solubilization efficiency and lower viscosity, a PPC concentration of $40 \mathrm{mg} / \mathrm{mL}$ was chosen for further investigations. At this concentration, the PPC-SDC molar ratio was 1 , which agrees with the results regarding the influence of the SDC concentration.

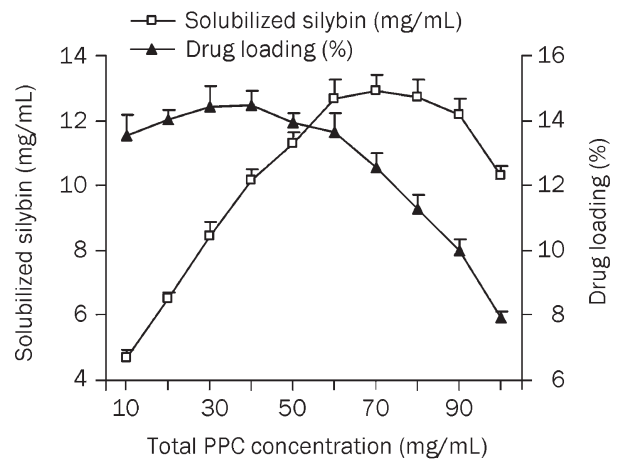

Figure 3. Influence of total PPC concentration on SLB solubility and drug loading at $10^{\circ} \mathrm{C}(\mathrm{SDC}$ conc, $20 \mathrm{mg} / \mathrm{mL})(n=3)$.

The effect of the $\mathrm{pH}$ value on the solubility of SLB in PPCSDC-MMs is shown in Figure 4. The results showed that enhancing the $\mathrm{pH}$ value of the mixed micellar solvent can dramatically increase the solubility of SLB when the $\mathrm{pH}$ is between 2 and 8 . However, SLB was not stable at $\mathrm{pH}$ val-

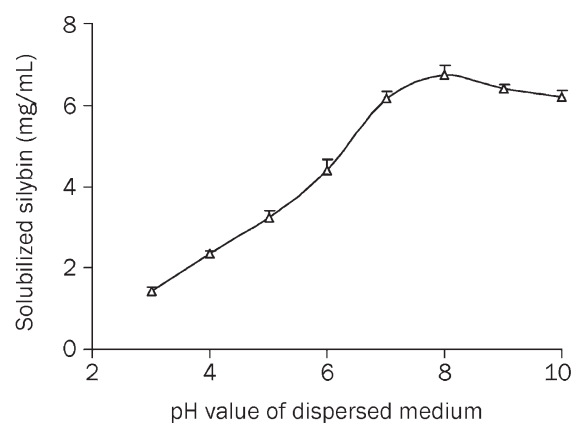

Figure 4. Effect of $\mathrm{pH}$ on SLB solubility in PPC-SDC-MMs at $10{ }^{\circ} \mathrm{C}(\mathrm{PPC} /$ $\mathrm{SDC}$ molar ratio=1; PPC conc $40 \mathrm{mg} / \mathrm{mL}$; dispersion medium: $0.05 \mathrm{~mol} / \mathrm{L}$ phosphate buffer) $(n=3)$. ues above 9 or even 8 . Hence, the optimum condition was between $\mathrm{pH} 7$ and 8 , which was exactly the $\mathrm{pH}$ value of the mixed micelles. Therefore, it was not necessary to use an additional $\mathrm{pH}$ regulator in this delivery system.

Other factors that affect the solubility of mixed micelles, such as ionic strength and temperature, were investigated, and the results are shown in Table 1. A rapid decrease in the solubility of silybin occurred with increasing ionic strength and temperature. Therefore, a low ionic strength and temperature are recommended for the preparation of SLB-PPC-SDC-MMs.

Table 1. Effect of ionic strength and temperature on silybin solubility in PPC-SDC-MMs (PPC/SDC molar ratio=1; PPC conc 40mg/mL) $(n=3)$.

\begin{tabular}{clc}
\hline $\begin{array}{c}\text { Temperature } \\
\left({ }^{\circ} \mathrm{C}\right)\end{array}$ & \multicolumn{1}{c}{ Dispersion medium } & $\begin{array}{c}\text { SLB solubility } \\
(\mathrm{mg} / \mathrm{mL})\end{array}$ \\
\hline 10 & Water & $10.34 \pm 0.29$ \\
10 & Phosphate buffer, $\mathrm{pH} 7.4,25 \mathrm{mmol} / \mathrm{L}$ & $8.73 \pm 0.37$ \\
10 & Phosphate buffer, $\mathrm{pH} 7.4,50 \mathrm{mmol} / \mathrm{L}$ & $6.46 \pm 0.31$ \\
25 & Water & $9.57 \pm 0.24$ \\
40 & Water & $8.22 \pm 0.25$ \\
\hline
\end{tabular}

\section{Particle size and morphology of SLB-PPC-SDC-MMs}

SLB-PPC-SDC-MMs were characterized by PCS and exhibited an average diameter of $(30.7 \pm 4.8) \mathrm{nm}$. SEM and TEM images of SLB-PPC-SDC-MMs are presented in Figures 5 and 6 , respectively. Both techniques confirmed that the mixed micelles were spherical in shape with a diameter of about 30 $\mathrm{nm}$.

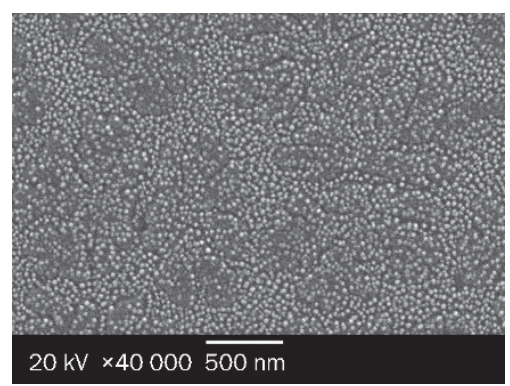

Figure 5. Scanning electron micrographs of SLB-PPC-SDC-MMs at $\times 40000$ magnification; scale bar: $500 \mathrm{~nm}$.

\section{Zeta potential}

Zeta potential is an important factor in evaluating the stability of a colloidal dispersion ${ }^{[25]}$. Generally speaking, the particles were stable when the absolute value of the zeta potential was above $30 \mathrm{mV}$ due to the electric repulsion between particles $^{[26-28]}$. The average zeta potential of the SLB-PPCSDC-MMs was $(-39 \pm 5.0) \mathrm{mV}$, which suggests that the MMs obtained in this study could be a dynamic stable system. 


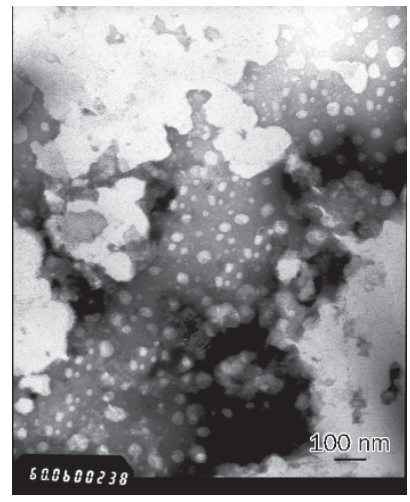

Figure 6. Transmission electron micrographs of SLB-PPC-SDC-MMs at $\times 60000$ magnification; scale bar: $100 \mathrm{~nm}$.

\section{In vivo studies in rats}

As seen in Figure 7, the internal standard (andrographolide) and silybin in plasma were completely separated under our analytical conditions, with retention time of 5 and $7 \mathrm{~min}$, respectively. Figure 8 and Table 2 show the main pharmacokinetic parameters in rats after intravenous injection of silybin$N$-methylglucamine or SLB-PPC-SDC-MMs (equivalent to 80 $\mathrm{mg} / \mathrm{kg}$ of silybin), respectively $(n=5)$. From the profile, we could see the mean retention time (MRT) and $\mathrm{AUC}_{(0-\infty)}$ of the SLB-PPC-SDC-MMs group were $29.8 \mathrm{~min}$ and $2190 \mathrm{mg} / \mathrm{L} \cdot \mathrm{min}$, respectively. In comparison, the $\mathrm{MRT}$ and $\mathrm{AUC}_{(0-\infty)}$ of the silybin-N-methylglucamine group were $14.8 \mathrm{~min}$ and 1517 $\mathrm{mg} / \mathrm{L} \cdot \mathrm{min}$, respectively, which were significantly lower $(P<0.05)$ than that of the SLB-PPC-SDC-MMs group. However, these two groups achieved approximately the same $C_{\max }$ value $(P>0.05)$. These results suggested the SLB-PPC-SDC$\mathrm{MMs}$ could remain in circulation in vivo longer than silybin$\mathrm{N}$-methylglucamine, which results in a higher $\mathrm{AUC}_{(0-\infty)}(1.44-$ fold).

\section{Discussion}

The phosphatidylcholine-bile salts-mixed micelle system has demonstrated great potential as a novel delivery vehicle for
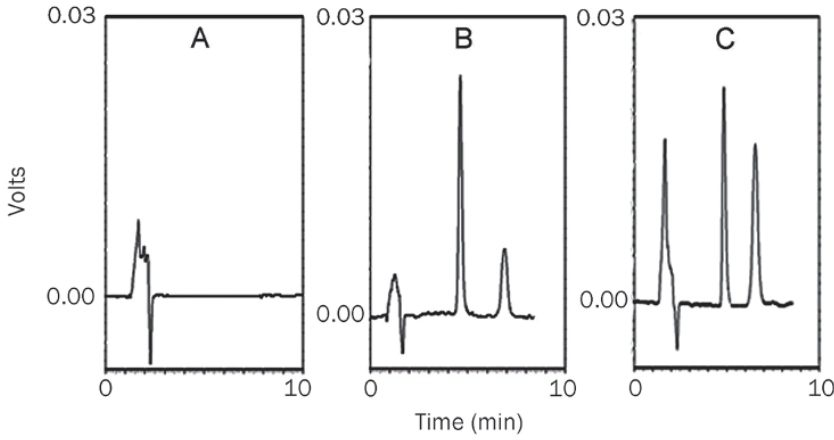

Figure 7. Typical chromatograms of silybin. (A) Blank rat plasma; (B) Blank rat plasma spiked with internal standard (andrographolide) and silybin; (C) A sample after intravenous administration of the SLB-PPC-SDCMMs.

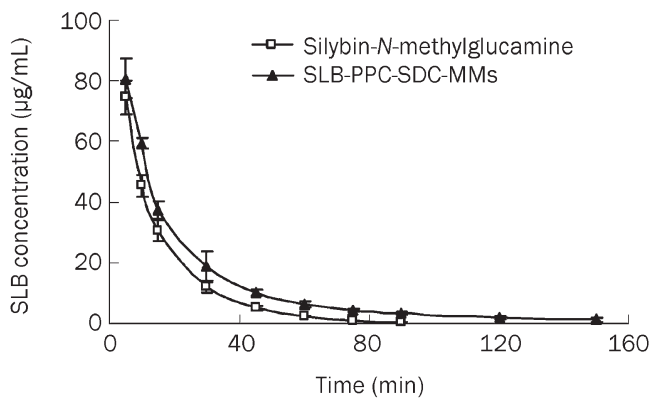

Figure 8. Mean plasma concentration-time curve of silybin in rats after intravenous administration of silybin- $N$-methylglucamine and SLB-PPCSDC-MMs $(n=5)$.

Table 2. The main pharmacokinetic parameters of silybin- $N$-methylglucamine and SLB-PPC-SDC-MMs in rats $(n=5)$.

\begin{tabular}{lccc}
\hline \multicolumn{1}{c}{ Parameters } & $\begin{array}{c}\text { Silybin- } N \text { - } \\
\text { methylglucamine }\end{array}$ & $\begin{array}{c}\text { SLB-PPC- } \\
\text { SDC-MMs }\end{array}$ & $t$-test \\
\hline $\mathrm{AUC}_{(0-\mathrm{t})}(\mathrm{mg} / \mathrm{L} \cdot \mathrm{min})$ & $1508 \pm 131$ & $2128 \pm 234$ & $P<0.05$ \\
$\mathrm{AUC}_{(0-\infty)}(\mathrm{mg} / \mathrm{L} \cdot \mathrm{min})$ & $1517 \pm 130$ & $2191 \pm 266$ & $P<0.05$ \\
$t_{1 / 2}(\mathrm{~min})$ & $13.0 \pm 0.83$ & $36.1 \pm 6.61$ & $P<0.05$ \\
$\mathrm{MRT}_{(0-\mathrm{t})}(\mathrm{min})$ & $14.2 \pm 0.18$ & $25.0 \pm 1.57$ & $P<0.05$ \\
$\mathrm{MRT}_{(0-\infty)}(\mathrm{min})$ & $14.8 \pm 0.11$ & $29.8 \pm 3.79$ & $P<0.05$ \\
$C_{\text {max }}(\mathrm{mg} / \mathrm{L})$ & $74.6 \pm 5.66$ & $80.7 \pm 6.47$ & $P>0.05$ \\
$\operatorname{Relative~bioavailability~(\% )~}$ & & 144 & \\
\hline
\end{tabular}

parenteral administration to overcome the obstacles of inadequate aqueous solubility and oral bioavailability. However, researchers are facing challenges associated with encapsulating drug substances that are neither lipophilic nor hydrophilic into such carriers. The development of a drug-phospholipid complex is expected to overcome this problem. In the current study, the complex greatly enhanced the lipophilicity of the drug substances, which proved to be well incorporated in the mixed micelles. Additionally, polyene phosphatidylcholine, the phospholipid we employed in the complexation process, has the same biological effect as SLB in maintaining liver health and treating liver disorders ${ }^{[2]}$. This suggests that the use of a SLB-PPC complex is a promising strategy for the systemic delivery of SLB.

In order to optimize the SLB-PPC-SDC-MM preparation, different process variables were evaluated, and the water-solubility and drug loading efficiency were employed as evaluation parameters. First, the influence of SDC concentration was investigated, and the results are shown in Figure 2. An increase of SDC concentration of up to $20 \mathrm{mg} / \mathrm{mL}$ resulted in a significant enhancement of drug solubility and drug loading efficiency. However, a further increase of SDC concentration led to dramatic reduction in drug loading efficiency, although SDC could still solubilize the drug to some extent. This underlying process accounts for achievement of the highest combination rate of SDC and PPC when the SDC concentration was 
increased to $20 \mathrm{mg} / \mathrm{mL}$. Although excess free SDC micelles could also solubilize SLB, the solubilizing capacity was weaker than that of the mixed micelles.

Another important factor in the SLB-PPC-SDC-MM formulation was PPC concentration. When the molar ratio of PPC/ SDC was below 1.0 (PPC concentration at $40 \mathrm{mg} / \mathrm{mL}$ ), the increase in solubility and drug loading efficiency of SLB was dramatic, which was due to the dual increase of both the size and the lipophilicity of PPC-SDC-MM upon the incorporation of $\mathrm{PPC}^{[19]}$. Continuously increasing the PPC concentration resulted in a relatively stable solubilization of SLB and a rapid decrease in drug loading efficiency. This was also because the strongest binding force between PPC and SDC was achieved, similar to the influence of the SDC concentration. In addition, an increase in the PPC concentration resulted in a higher viscosity and, consequently, less partition of the drug into the mixed micellar space.

As for the effect of $\mathrm{pH}$ on the solubility of SLB, the results showed that the optimum condition for the SLB-PPC-SDCMMs was between $\mathrm{pH} 7$ and 8 . This could be attributed to the different surface charges of the mixed micelles under different conditions. The lipid carrier displayed a less negative zeta potential at $\mathrm{pH} 2$ than $\mathrm{pH} 8$ and led to aggregation and further precipitation of the mixed micelles at lower $\mathrm{pH}$ values, which resulted in less solubilization of SLB under these conditions. However, some interactions between silybin and phospholipids, such as the combination of hydrogen bonds or van der Waals forces ${ }^{[13,30,31]}$, may not be very stable in highly charged environments (above $\mathrm{pH} 8$ ). Therefore, a moderate $\mathrm{pH}$ value of medium is recommended for the preparation of the SLBPPC-SDC-MMs.

Other factors such as increased ionic strength and temperature resulted in a rapid decrease in the solubility of silybin. This may be due to an increased critical micelle concentration under those conditions. Consequently, fewer micelles were available for the solubilization process. These findings are in good agreement with previous reports ${ }^{[2,33]}$.

After different parameters were screened to obtain the optimized formulation, some characteristics of the SLB-PPC-SDCMMs were investigated, such as particle size, zeta potential and morphology. The results showed the particles were $30 \mathrm{~nm},-39 \mathrm{mV}$ and spherical in shape, indicating it was a dynamic, stable and uniform system. Hence, it was necessary to develop a schematic representation of the structure of this formulation.

As mentioned in the identification of the SLB-PPC complex by DSC, hydrogen bonding played a major role in SLB-PPC complexation $^{[13-15,31]}$; the $-\mathrm{OH}$ groups of the phenol rings of SLB were engaged in hydrogen bonding with the phospholipids. This indicated that it was the polar part of PPC that interacted with the silybin. The structure of the PC-BS-MMs, studied systematically by many researchers ${ }^{[19,34]}$, is widely

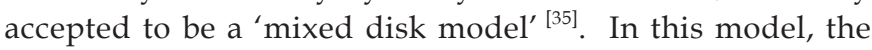
mixed micelle consists of a disk-like portion of a phospholipid bilayer surrounded at its perimeter by bile salt molecules, exposing their hydrophilic surfaces to the aqueous solvent and their lipophilic surfaces to the hydrocarbon tails of the phospholipid molecules. However, our observations by SEM and TEM indicated that the SLB-PPC-SDC-MMs were spherical in shape, which may be due to the presence of the SLBPPC complex. Therefore, based on our experimental results, a schematic representation of the structure of the SLB-PPC-SDCMMs is presented in Figure 9. Silybin first combines with the polar head group of PPC, forming a SLB-PPC complex. Then, using the co-precipitation method, the hydrocarbon tails of the PPC bind to the hydrophobic surface of SDC, forming spheres and exposing the hydrophilic surfaces of SDC to contact with the aqueous solvent.

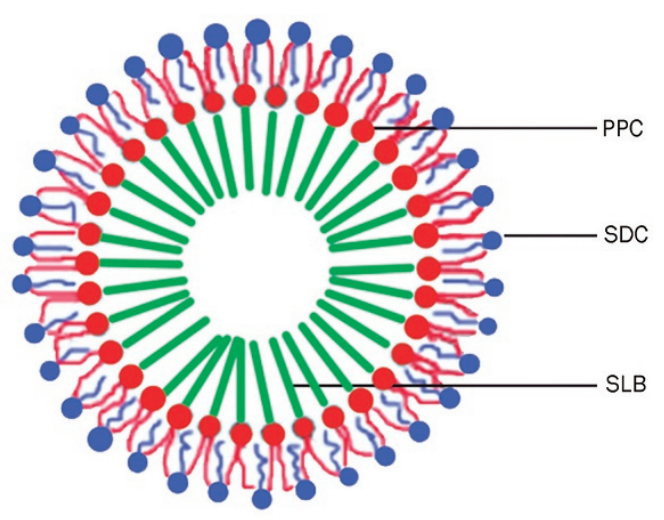

Figure 9. Schematic representation of the structure of the SLB-PPC-SDCMMs.

To further understand the characteristics of the SLB-PPCSDC-MMs in vivo, pharmacokinetic studies were also performed. The results showed that the SLB-PPC-SDC-MMs remained in circulation in vivo longer than silybin- $N$-methylglucamine, which resulted in a higher AUC. Theoretically, the increased MRT and AUC were attributed to interactions between the drug and PPC-SDC-MMs when the mixed micelles were above the $\mathrm{CMC}^{[36,37]}$ at the beginning of administration. These combined forces would prolong the release of SLB into the blood. However, once the mixed micelles were diluted below the critical micelle concentration, the entrapped drug would be released into circulation. At this time, the released SLB-PPC complex, with different lipophilicity and solubility behavior, would play a major role in enhancing the MRT and AUC of SLB in vivo. This assumption is also verified by other literature ${ }^{[15]}$, which found that oral administration of a SLB-phospholipid complex could greatly enhance the MRT and AUC of SLB. In conclusion, it was the PPC-SDC-MMs and SLB-PPC complex combined that influenced the behavior of SLB in vivo.

Therefore, the SLB-PPC complex could not only improve the lipophilicity of SLB to be better enveloped in the PPC-SDCMMs, but also increase its circulation time in vivo. The mixed micelle system, which remarkably improved the solubility of the SLB-PPC complex in water, could be used as a formulation for intravenous administration. PPC-SDC-MMs have been 
investigated for the oral administration of silybin ${ }^{[38]}$. Nevertheless, we encapsulated the SLB-PPC complex in this carrier for intravenous administration because the oral bioavailability of SLB in rats was estimated to only be $0.73 \%^{[12]}$. To maximize the drug efficacy, it was necessary to develop a delivery system that can directly distribute into each organ, thereby avoiding the absorption process. Therefore, the obtained SLB-PPCSDC-MMs in the current study should provide a dramatic increase in bioavailability compared to oral administration.

\section{Conclusions}

In this paper, we have shown that silybin, a poorly soluble behavior drug, can be successfully enveloped into PPC-SDCMMs after the formation of an SLB-PPC complex. The novel formulation with optimized formulation parameters dramatically improved both the water-solubility and the mean retention time of the drug in vivo. As a result, a new pattern of mixed micelles loaded with a phospholipid complex was proposed. This novel strategy was believed to be attractive for the development of drug substances that exhibit poor solubility.

\section{Acknowledgements}

Financial support of this research was provided by the National Science \& Technology Major Project of China (grant № 2009ZX09310-002) and the National Natural Science Foundation of China (№ 30873165).

The authors thank Zhi-xiang YUAN, San-jun SHI and Chang-tao LUAN for their help with these experiments.

\section{Author contribution}

Zhi-rong ZHANG and Tao GONG designed the research; Ruiling DUAN performed the research and wrote the paper; Xun SUN and Jie LIU revised the paper.

\section{References}

1 Dressman J, Reppas C. Drug solubility: how to measure it, how to improve it. Adv Drug Deliver Rev 2007; 59: 531-2.

2 Serajuddin, Abu TM. Salt formation to improve drug solubility. Adv Drug Deliver Rev 2007; 59: 603-16.

3 Blagden N, Matas M de, Gavan PT, York P. Crystal engineering of active pharmaceutical ingredients to improve solubility and dissolution rates. Adv Drug Deliver Rev 2007; 59: 617-30.

4 Kesisoglou F, Panmai S, Wu Y. Nanosizing - Oral formulation development and biopharmaceutical evaluation. Adv Drug Deliver Rev 2007; 59: 631-44.

5 Hauss DJ. Oral lipid-based formulations. Adv Drug Deliver Rev 2007; 59: 667-76.

6 Brewster ME, Loftsson T. Cyclodextrins as pharmaceutical solubilizers. Adv Drug Deliver Rev 2007; 59: 645-66.

7 Stella VJ, Nti-Addae KW. Prodrug strategies to overcome poor water solubility. Adv Drug Deliver Rev 2007; 59: 677-94.

8 Gao Y, Li LB, Zhai G. Preparation and characterization of Pluronic/ TPGS mixed micelles for solubilization of camptothecin. Colloid Surface B 2008; 64: 194-9.

9 Kvasnicka F, Biba B, Sevcik R, Voldrich M, Kratka J. Analysis of the active components of silymarin. J Chromatogr A 2003; 990: 239-45.

10 Tedesco D, Tava A, Galletti S, Tameni M, Varisco G, Costa A, et al.
Effects of silymarin, a natural hepatoprotector, in periparturient dairy cows. J Dairy Sci 2004; 87: 2239-47.

11 Trappoliere M, Caligiuri A, Schmid M, Bertolani C, Failli P, Di Manzano C, et al. Silybin is a direct antifibrogenic and antiinflammatory agent: Cellular and molecular mechanisms. Digest Liver Dis 2008; 40: A127.

12 Wu JW, Lin LC, Hung SC, Chi CW, Tsai TH. Analysis of silibinin in rat plasma and bile for hepatobiliary excretion and oral bioavailability application. J Pharmaceut Biomed 2007; 45: 635-41.

13 Lasonder E, Weringa WD. An NMR and DSC study of the interaction of phospholipids vesicles with some anti-inflammatory agents. J Colloid Interf Sci 1990; 139: 469-78.

14 Morazzoni P, Magistretti MJ, Giachetti C, Zanolo G. Comparative bioavailability of silipide, a new flavanolignan complex, in rats. Euro J Drug Metab Pharmacokinet 1992; 17: 39-44.

15 Xiao YY, Song YM, Chen ZP, Ping QN. The preparation of silybin phospholipid complex and the study on its pharmacokinetics in rats. Int J Pharm 2006; 307: 77-82.

16 Chen W, Xia H, Wu W. Optimized preparation of silymarin dripping pills by a central composite design-response surface method. Chin Trad Herb Drug 2005; 36: 679-83.

17 Wu W, Wang Y, Que L. Enhanced bioavailability of silymarin by selfmicroemulsifying drug delivery system. Eur J Pharm Biopharm 2006; 63: 288-94.

18 Alkan-Onyuksel H, Ramakrishnan S, Chai HB, Pezzuto JM. A mixed micellar formulation suitable for the parenteral administration of taxol. Pharm Res 1994; 11: 206-12.

19 Hammad MA, Müller BW. Increasing drug solubility by of bile saltphosphatidylcholine-based mixed micelles. Eur J Pharm Biopharm 1998; 46: 361-7.

20 Hammad MA, Müller BW. Solubility and stability of tetrazepam in mixed micelles. Eur J Pharm Sci 1998; 7: 49-55.

21 Steffen H. Prinzip Mischmizelle. Die Gallensäure-Lecithin-Mischmizllen als Trägersystem schwer wasserlösliche Wirkstoffe. Roche Magazine 1984; 20: 2-9.

22 Teelmann K, Schläppi B, Schüpbach M, Kistler A. Preclinical safety evaluation of intravenously administered mixed micelles. Arzneimittelforschung 1984; 34: 1517-23.

23 Schurtenberger P, Mazer N, Känzig W. Miceller vesicle transition in aqueous solution of bile salt and lecithin. J Phys Chem 1985; 89: 1042-9.

24 Maiti K, Mukherjee K, Gantait A, Saha BP, Mukherjee PK. Curcuminphospholipid complex: Preparation, therapeutic evaluation and pharmacokinetic study in rats. Int J Pharm 2007; 330: 155-63.

25 Komatsu H, Kitajima A, Okada S. Pharmaceutical characterzation of commercially available intravenous fat emulsions: estimation of average particle size, size distribution and surface potential using photon correlation spectroscopy. Chem Pharm Bull 1995; 43: 14125.

26 Müller RH, Mäder K, Gohla S. Solid lipid nanoparticles (SLN) for controlled drug delivery - a review of the state of the art. Eur J Pharm Biopharm 2000; 50: 161-77.

27 Müller RH, Rühl D, Runge SA. Biodegradation of solid lipid nanoparticles as a function of lipase incubation time. Int J Pharm 1996; 144: 115-21.

28 Liu J, Gong T, Wang CG, Zhong ZR, Zhang ZR. Solid lipid nanoparticles loaded with insulin by sodium cholate-phosphatidylcholine-based mixed micelles: Preparation and characterization. Int J Pharm 2007; 340: $153-62$.

29 Horejsová M, Urban J. The effect of polyene phosphatidylcholine (Essentiale forte) in the treatment of liver steatosis and ultrasound findings--preliminary study. Cas Lek Cesk 1994; 133: 366-9. 
30 Hwang SB, Shen TY. Membrane effects of anti-inflammatory agents. 2. Interaction of nonsteroidal anti-inflammatory drugs with liposome and purple membranes. J Med Chem 1981; 24: 1202-12.

31 Venema FR, Weringa WD. The interactions of phospholipid vesicles with some anti-inflammatory agents. J Colloid Interf Sci 1988; 125 : 484-500.

32 Flockhart BD. The effect of temperature on the critical micelle concentration of some paraffin-chain salt. J Colloid Sci 1961; 16: 484-92.

33 Alkan-Onyuksel H, Son K. Mixed micelles as proliposomes for the solubilization of tenposide. Pharm Res 1992; 9: 1556-62.

34 Carey MC, Small DM. The characteristics of mixed micellar solutions with particular reference to bile. Amer J Med 1970; 49: 590-608.
35 Mazer NA, Benedek GB, Carey MC. Quasielastic light-scattering studies of aqueous biliary lipid systems. Mixed micelle formation in bile salt-lecithin solutions. Biochem 1980; 19: 601-15.

36 Dongowski G, Fritzscb B, Giessler J, Härtl A, Kuhlmann O, Neuber $\mathrm{RHH}$. The influence of bile salts and mixed micelles on the pharmacokinetics of quinine in rabbits. Eur J Pharm Biopharm 2005; 60: 147-51.

37 Ploema FGJ, Tucker JJ, Crommelin DJA. The role of bile salts in the intestinal absorption of drugs. Acta Pharm Technol 1990; 36: 43-52.

38 Yu JN, Zhu Y, Wang L, Peng M, Tong SS, Cao X, et al. Enhancement of oral bioavailability of the poorly water-soluble drug silybin by sodium cholate/phospholipid-mixed micelles. Acta Pharmacol Sin 2010; 31: 759-64. 